\title{
FINITE-DIFFERENCE PRECONDITIONERS FOR SUPERCONSISTENT PSEUDOSPECTRAL APPROXIMATIONS
}

\author{
Lorella Fatone $^{1}$, Daniele Funaro ${ }^{1}$ and Valentina Scannavini ${ }^{1}$
}

\begin{abstract}
The superconsistent collocation method, which is based on a collocation grid different from the one used to represent the solution, has proven to be very accurate in the resolution of various functional equations. Excellent results can be also obtained for what concerns preconditioning. Some analysis and numerous experiments, regarding the use of finite-differences preconditioners, for matrices arising from pseudospectral approximations of advection-diffusion boundary value problems, are presented and discussed, both in the case of Legendre and Chebyshev representation nodes.
\end{abstract}

Mathematics Subject Classification. 65N35, 65F15, 41A10.

Received July 3, 2007.

\section{INTRODUCTION}

Given a linear differential operator $L$ and a function $f$, we look for spectral type algebraic polynomial approximations to the solution $u$ of the problem

$$
L u=f \quad \text { in } \quad]-1,1[\text {. }
$$

For simplicity, we assume homogeneous Dirichlet boundary conditions, although other kind of boundary constraints can be treated without any problem.

We would like to approximate the solution of (1) by a superconsistent collocation method (see $[6,8,9]$ ). This means that we need two sets of nodes for this discretization. We call the first set of nodes, belonging to ]-1,1[, the representation grid. They are denoted by $\mathbf{x}=\left\{x_{j}\right\}_{j=1, \ldots, n-1}$, where $n$ is the discretization parameter. This grid is actually used to represent the approximated solution $u_{n}$, belonging to $\mathbb{P}_{n}^{0}$, which is the space of polynomials of degree less than or equal to $n$, vanishing at $x= \pm 1$. Due to this, the polynomial $u_{n}$ will be regarded as a linear combination of the Lagrange basis functions $l_{j} \in \mathbb{P}_{n}^{0}, j=1, \ldots, n-1$, with respect to the set of nodes $\mathbf{x}$, that is:

$$
u_{n}=\sum_{j=1}^{n-1} u_{n}\left(x_{j}\right) l_{j}, \quad \text { with } \quad l_{j}\left(x_{i}\right)=\delta_{i j}, \quad i, j=1, \ldots, n-1,
$$

where $\delta_{i j}$ is the Kronecker symbol.

\footnotetext{
Keywords and phrases. Spectral collocation method, preconditioning, superconsistency, Lebesgue constant.

1 Dipartimento di Matematica Pura ed Applicata, via Campi 213/b, Università di Modena e Reggio Emilia, Modena 41110, Italy. lorella.fatone@unimo.it; daniele.funaro@unimo.it; s.valentina@tin.it
} 
The second set of nodes, belonging to ]-1,1[, is called collocation grid (or residual grid) and denoted by $\mathbf{z}=\left\{z_{i}\right\}_{i=1, \ldots, n-1}$. The discretized operator $\mathbf{L}_{n}$ is a vector map defined in the following way. For a function $v$, the $i$-th component of $\mathbf{L}_{n} v$ is given by:

$$
\left\{\mathbf{L}_{n} v\right\}_{i}=\sum_{j=1}^{n-1} v\left(x_{j}\right)\left(L l_{j}\right)\left(z_{i}\right)
$$

We expect that $\left\{\mathbf{L}_{n} v\right\}_{i}$ is an approximation of $(L v)\left(z_{i}\right)$. Indeed, every time $v$ belongs to $\mathbb{P}_{n}^{0}$, it is true that:

$$
\left\{\mathbf{L}_{n} v\right\}_{i}-(L v)\left(z_{i}\right)=0, \quad i=1, \ldots, n-1,
$$

since $v$ can be written as $v=\sum_{j=1}^{n-1} v\left(x_{j}\right) l_{j}$. Note that, in vector form, $(3)$ is equivalent to write:

$$
\mathbf{L}_{n} v-(L v)(\mathbf{z})=0
$$

The discrete solution $u_{n}$ will be then required to satisfy the following equation:

$$
\mathbf{L}_{n} u_{n}=f(\mathbf{z})
$$

from where it can be easily deduced a linear system of dimension $n-1$, having $u_{n}(\mathbf{x})$ as unknown vector.

Usually, the two grids $\mathbf{x}$ and $\mathbf{z}$ coincide and are related to zeros of orthogonal polynomials, such as Legendre or Chebyshev polynomials, or, more appropriately, to the zeros of their derivatives. However, we are free to choose collocation nodes which are different from the representation nodes. Actually, in the superconsistent method, we are concerned with finding, for a given set of nodes $\mathbf{x}$, a new set of nodes $\mathbf{z}$ in such a way that (4) is true also for functions that do not belong to $\mathbb{P}_{n}^{0}$. For instance, we want (4) to be also satisfied for all functions $v$ which are polynomials of degree $n+1$, vanishing at $x= \pm 1$, that is $v \in \mathbb{P}_{n+1}^{0}$. We see later in the paper how to build up the collocation grid in order to achieve such a superconsistency property.

There are several advantages in using collocation nodes linked to representation nodes in a superconsistent way. First of all, we improve the numerical performances of the method. The improvement is mild concerning symmetric type differential operators, such as $L=-\frac{\mathrm{d}^{2}}{\mathrm{~d} x^{2}}$, but it is extremely significant in the case of the advection-dominated operator $L=-\epsilon \frac{\mathrm{d}^{2}}{\mathrm{~d} x^{2}}+\beta \frac{\mathrm{d}}{\mathrm{d} x}$ (see [7]). Many of the results of this paper will deal with the treatment of this non symmetric case, which is known to be quite nasty when the diffusion coefficient $\epsilon$ is very small compared to the transported coefficient $\beta$.

As well explained in the survey paper by Mund [17], another important feature is the possibility of constructing excellent finite-difference preconditioners, especially in the non symmetric case, where in the classical collocation method this technique does not work properly if the ratio $\beta / \epsilon$ is large (see $[14,15]$ ). The most of the paper will be devoted to this aspect, both for approximations based on Legendre and Chebyshev representation nodes. Together with some theory and a series of numerical experiments, we shall point out several remarkable properties that can be useful for other applications and extensions. Note that, although the superconsistent method is very effective, very little theory has been developed up to now. This paper, unfortunately restricted to one-dimensional case, is a first attempt towards a rigorous analysis.

\section{Preconditioners For the SECONd DeRIVATIVe operator in the Legendre CASE}

Let $L$ in (1) be the second derivative operator: i.e. $L=-\frac{\mathrm{d}^{2}}{\mathrm{~d} x^{2}}$. Thus, for a given function $f$ we consider the following Dirichlet boundary-value problem:

$$
\left.-u^{\prime \prime}=f \quad \text { in }\right]-1,1[, \quad \text { with } \quad u(-1)=u(1)=0 .
$$

A collocation method to approximate $u$ can be constructed by taking the points of the grid $\mathbf{x}$ to be the zeros of $P_{n}^{\prime}$, where $P_{n}$ is the Legendre polynomial of degree $n$. In the literature these nodes are known as Legendre 
Gauss-Lobatto nodes, but here, for brevity, we refer to them as Legendre nodes. Then, we look for $u_{n} \in \mathbb{P}_{n}^{0}$ such that:

$$
-u_{n}^{\prime \prime}\left(x_{i}\right)=f\left(x_{i}\right) \quad \text { for } \quad i=1, \ldots, n-1 .
$$

It is known (see [2]) that $u_{n}$ converges to $u$ for $n \rightarrow+\infty$, and that the rate of convergence is of spectral type, that is, it only depends on the regularity of $u$.

We can now introduce another collocation grid $\mathbf{z}$, and consider the problem of finding a new solution $w_{n} \in \mathbb{P}_{n}^{0}$ such that:

$$
-w_{n}^{\prime \prime}\left(z_{i}\right)=f\left(z_{i}\right) \quad \text { for } \quad i=1, \ldots, n-1 .
$$

As we already mentioned, let us note that (4) is automatically satisfied for any $v \in \mathbb{P}_{n}^{0}$, whatever is the grid $\mathbf{z}$. In order to have superconsistency we have to require that (4) is satisfied also for $v \in \mathbb{P}_{n+1}^{0}$. This means that $\mathbf{z}$ must be in such a way that:

$$
\sum_{j=1}^{n-1} v\left(x_{j}\right) l_{j}^{\prime \prime}\left(z_{i}\right)-v^{\prime \prime}\left(z_{i}\right)=0, \quad i=1, \ldots, n-1,
$$

for all polynomials $v \in \mathbb{P}_{n+1}^{0}$. To find $\mathbf{z}$ we start by noting that any polynomial $v$ of degree $n+1$, vanishing at $x= \pm 1$, can be written as

$$
v=c \chi_{n+1}+q_{n}
$$

where $c$ is a constant, $\chi_{n+1}=\left(1-x^{2}\right) P_{n}^{\prime}$ belongs to $\mathbb{P}_{n+1}^{0}$, and $q_{n}$ belongs to $\mathbb{P}_{n}^{0}$. Hence, by linearity, the following non linear equation for the unknown $\mathbf{z}$ must hold:

$$
\mathbf{L}_{n} \chi_{n+1}-\left(L \chi_{n+1}\right)(\mathbf{z})=0 .
$$

Since $\chi_{n+1}(\mathbf{x})=0$, according to (2), we discover that $\mathbf{L}_{n} \chi_{n+1}=0$. Therefore, each $z_{i}$ must be solution to the equation:

which has exactly $n-1$ zeros.

$$
\left(L \chi_{n+1}\right)\left(z_{i}\right)=-\chi_{n+1}^{\prime \prime}\left(z_{i}\right)=0
$$

We can say more about these zeros by using the following Sturm-Liouville problem:

$$
-\left(1-x^{2}\right) P_{n}^{\prime \prime}+2 x P_{n}^{\prime}=n(n+1) P_{n},
$$

that characterizes the family of Legendre polynomials. From (10) we have:

$$
-\chi_{n+1}^{\prime \prime}\left(z_{i}\right)=-\left[\left(1-x^{2}\right) P_{n}^{\prime}\right]^{\prime}\left(z_{i}\right)=n(n+1) P_{n}^{\prime}\left(z_{i}\right)=0, \quad i=1, \ldots, n-1 .
$$

In other words, we find out that $\mathbf{x}$ should be the same as $\mathbf{z}$, that shows that the collocation problem (6) is already superconsistent. Therefore, we must have: $w_{n}=u_{n}$. This is however a very special case. As we shall see later on, when $L$ is a different operator (see Sect. 5) or when the nodes $\mathbf{x}$ are zeros of other sets of orthogonal polynomials (see Sect. 2), the representation grid and the superconsistent collocation grid do not coincide.

An interesting result is the following one.

Theorem 1.1. Let $L=-\frac{\mathrm{d}^{2}}{\mathrm{~d} x^{2}}$, with homogeneous Dirichlet boundary conditions in $x= \pm 1$. Suppose that (4) is true for any $v \in \mathbb{P}_{n+1}^{0}$. Then $\mathbf{x}=\mathbf{z}$ if and only if the points $x_{i}, i=1, \ldots, n-1$, are the zeros of $P_{n}^{\prime}$.

Proof. We have already shown that if $\mathbf{x}$ is such that $P_{n}^{\prime}\left(x_{i}\right)=0, i=1, \ldots, n-1$, then $\mathbf{z}=\mathbf{x}$. To prove the converse, let us assume that $\mathbf{z}=\mathbf{x}$ and that $Q_{n}$ is a polynomial of degree $n$ such that $Q_{n}^{\prime}\left(x_{i}\right)=0$, $i=1, \ldots, n-1$. Then $v=\left(1-x^{2}\right) Q_{n}^{\prime} \in \mathbb{P}_{n+1}^{0}$, so that, due to (8), we must have $v^{\prime \prime}\left(z_{i}\right)=v^{\prime \prime}\left(x_{i}\right)=0$. This says that $Q_{n}^{\prime}$ and $v^{\prime \prime}$, which have the same degree, have common roots. Therefore, they are proportional, i.e. $v^{\prime \prime}=\left[\left(1-x^{2}\right) Q_{n}^{\prime}\right]^{\prime \prime}=\lambda Q_{n}^{\prime}$. Integrating, we have:

$$
\left[\left(1-x^{2}\right) Q_{n}^{\prime}\right]^{\prime}=\lambda Q_{n}+c,
$$


where $c$ is a constant. If we look for polynomial solutions, we end up with the set of Legendre polynomials (see, for instance [5]). Then, it is easy to check that there must be two constants $a$ and $b$ such that $Q_{n}=a P_{n}+b$. Hence, the nodes $\mathbf{x}$ are the roots of $P_{n}^{\prime}$.

We are now concerned with finding the entries of the discretization matrix

$$
A_{n-1}=\left\{-l_{j}^{\prime \prime}\left(x_{i}\right)\right\}_{i, j=1, \ldots, n-1},
$$

associated to the linear system arising from (6).

We first give the expression of the polynomials of the Lagrange basis (see, for instance, [10]):

$$
l_{j}(x)=-\frac{1}{n(n+1) P_{n}\left(x_{j}\right)} \frac{\left(1-x^{2}\right) P_{n}^{\prime}(x)}{x-x_{j}}, \quad j=1, \ldots, n-1 .
$$

Differentiating twice and evaluating at $\mathbf{x}$, we get:

$$
\begin{aligned}
& l_{j}^{\prime \prime}\left(x_{i}\right)=-\frac{P_{n}\left(x_{i}\right)}{P_{n}\left(x_{j}\right)} \frac{2}{\left(x_{i}-x_{j}\right)^{2}}, \quad i, j=1, \ldots, n-1, i \neq j, \\
& l_{i}^{\prime \prime}\left(x_{i}\right)=-\frac{n}{3\left(1-x_{i}^{2}\right)}, \quad i=1, \ldots, n-1 .
\end{aligned}
$$

Thus, the matrix $A_{n-1}$ is full and non symmetric. Moreover, it is known to have positive real eigenvalues, the maximum of them growing as $n^{4}$ when $n$ grows.

Due to the above considerations, the matrix is ill-conditioned. Hence, it is not suitable when the system is solved by an iterative method. In the past, very effective preconditioners were proposed (see $[1,11,17,18]$ ). It is sufficient, for example, to consider the matrix $M_{n-1}$, of dimension $n-1$, related to the finite-difference discretization of the operator $L=-\frac{\mathrm{d}^{2}}{\mathrm{~d} x^{2}}$.

For a given function $v$ vanishing at $x= \pm 1$, the $i$-th component, $i=1, \ldots, n-1$, of the vector obtained by applying $M_{n-1}$ to the vector $v(\mathbf{x})$ is given by:

$$
\left\{M_{n-1} v(\mathbf{x})\right\}_{i}=\frac{-2}{x_{i+1}-x_{i-1}}\left[\frac{v\left(x_{i+1}\right)-v\left(x_{i}\right)}{x_{i+1}-x_{i}}-\frac{v\left(x_{i}\right)-v\left(x_{i-1}\right)}{x_{i}-x_{i-1}}\right],
$$

with $x_{0}=-1$ and $x_{n}=1$. It turns out that $M_{n-1}$ is tridiagonal.

If we compute the eigenvalues of $M_{n-1}^{-1} A_{n-1}$, we discover that they are all real and positive. Moreover, the minimum eigenvalue is always equal to 1 . This can be explained by noting that, for $n \geq 2$, there is no difference in applying the spectral operator or the finite-difference operator to the vector obtained by interpolating the second degree polynomial $1-x^{2}$ at the grid $\mathbf{x}$. Denoting by $\lambda_{n}$ the maximum eigenvalue of $M_{n-1}^{-1} A_{n-1}$, we find out that this is bounded independently of $n$ by a constant. The proof of this fact is provided in [14,15]. We report in Table 1, the values of $\lambda_{n}$ for various $n$.

The extension of these results in the case of the Laplace operator can be also found in [14] and detailed numerical experiments are reported in [7].

\section{Preconditioners for the Second derivative operator in the Chebyshev case}

Another collocation method for problem (5) is obtained by taking the points of the grid $\mathbf{x}$ to be the zeros of $T_{n}^{\prime}$, where $T_{n}$ is the Chebyshev polynomial of degree $n$. These zeros are known as Chebyshev Gauss-Lobatto nodes, and we briefly refer to them as Chebyshev nodes. We recall that, in this case, the exact expression of these zeros is known, i.e.:

$$
x_{j}=-\cos \theta_{j} \quad \text { where } \quad \theta_{j}=\frac{j \pi}{n}, \quad j=1, \ldots, n-1 .
$$


TABLE 1. Maximum preconditioned eigenvalues for the collocation method at the zeros of $P_{n}^{\prime}$.

\begin{tabular}{cccc}
\hline$n$ & $\lambda_{n}$ & $n$ & $\lambda_{n}$ \\
\hline 5 & 1.72 & 13 & 2.11 \\
6 & 1.81 & 14 & 2.13 \\
7 & 1.88 & 15 & 2.15 \\
8 & 1.93 & 16 & 2.17 \\
9 & 1.98 & 17 & 2.18 \\
10 & 2.02 & 18 & 2.20 \\
11 & 2.05 & 19 & 2.21 \\
12 & 2.08 & 20 & 2.26 \\
\hline
\end{tabular}

In addition, the Chebyshev polynomials satisfy the following Sturm-Liouville problem:

$$
\left(1-x^{2}\right) T_{n}^{\prime \prime}-x T_{n}^{\prime}+n^{2} T_{n}=0,
$$

from which, by differentiation, we obtain the two relations:

$$
\begin{aligned}
{\left[\left(1-x^{2}\right) T_{n}^{\prime}\right]^{\prime} } & =-\left(n^{2} T_{n}+x T_{n}^{\prime}\right) \\
{\left[\left(1-x^{2}\right) T_{n}^{\prime}\right]^{\prime \prime} } & =-\left[\left(n^{2}+1\right) T_{n}^{\prime}+x T_{n}^{\prime \prime}\right]
\end{aligned}
$$

In order to recover the new superconsistent grid $\mathbf{z}$, we proceed as in Section 1. We can easily deduce the equation (9), where now $\chi_{n+1}$ is given by $\chi_{n+1}=\left(1-x^{2}\right) T_{n}^{\prime}$. Taking into account (15), we can write:

$$
-\chi_{n+1}^{\prime \prime}\left(z_{i}\right)=0 \Leftrightarrow\left[\left(n^{2}+1\right) T_{n}^{\prime}\left(z_{i}\right)+z_{i} T_{n}^{\prime \prime}\left(z_{i}\right)\right]=0, \quad i=1, \ldots, n-1 .
$$

By virtue of Theorem 1.1 in Section 1, the grid $\mathbf{z}$ is now different from the grid $\mathbf{x}$. In [8], the following estimate regarding the location of the nodes $\mathbf{z}$ is given:

$$
\begin{cases}x_{j}<z_{j}<\xi_{j+1}, & j=1, \ldots,[n / 2], \\ \xi_{j}<z_{j}<x_{j}, & j=[n / 2]+1, \ldots, n-1, \\ z_{j}=0, & n \text { even and } j=n / 2,\end{cases}
$$

where $[*]$ is the integer part of $*$ and $\xi_{j}=-\cos \frac{(2 j-1) \pi}{2 n}, j=1, \ldots, n$. The computation of the superconsistent nodes is easily carried out using an iterative method combined with the recurrence formulas for Chebyshev polynomials. To this purpose, the bounds given in (17) are helpful for setting the initial guesses. For $n=10$, we show in Figure 1 the distribution of the nodes $x_{i}, i=1, \ldots, 9$ (black circles) and that of the superconsistent Chebyshev nodes $z_{i}, i=1, \ldots, 9$ (white circles). Due to the difference between the representation grid $\mathbf{x}$ and the collocation grid $\mathbf{z}$, the solution $u_{n}$ of problem (6) does not coincide with the solution $w_{n}$ of problem (7). In [8], some numerical tests show that, in the $L^{2}$ and $L^{\infty}$ norms, the error $\left|u-w_{n}\right|$ decay faster than the error $\left|u-u_{n}\right|$, and this last quantity is known to converge to zero exponentially. We discuss some convergence results in the next section. A complete theoretical convergence analysis of the discrete superconsistent Chebyshev solution $w_{n}$ to the exact solution $u$ for $n \rightarrow \infty$ can be found in [4]. Unfortunately, let us note that the error estimate proven in [4] is not optimal. However the study of the Lebesgue constants shown in Section 4 and the numerical experiments of Section 5 actually ensure an optimal decay of the error. This means that the theoretical results in [4] could be improved upon, although this kind of analysis looks quite nasty. 


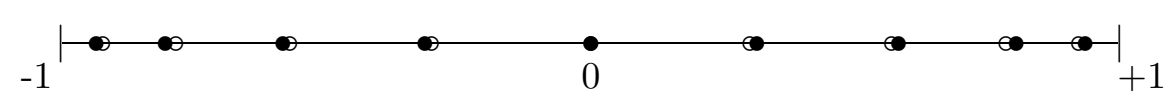

Figure 1. The grid $\mathbf{x}$ (black circles) and the grid $\mathbf{z}$ (white circles) for the superconsistent Chebyshev method with $n=10$.

The improvement in convergence is one of the reasons to prefer the superconsistent approach. Another reason emerges from the study of the preconditioned eigenvalues. As in Section 1, we denote by $A_{n-1}$ the spectral discretization matrix (11) associated with the Chebyshev nodes. On the other hand, we now have a new matrix $B_{n-1}$ :

$$
B_{n-1}=\left\{-l_{j}^{\prime \prime}\left(z_{i}\right)\right\}_{i, j=1, \ldots, n-1} .
$$

The polynomials of the Lagrange basis with respect to the grid $\mathbf{x}$ are given by:

$$
l_{j}(x)=-\frac{(-1)^{n+j}}{n^{2}} \frac{\left(1-x^{2}\right) T_{n}^{\prime}(x)}{x-x_{j}}, \quad j=1, \ldots, n-1 .
$$

Differentiating twice and evaluating at $\mathbf{x}$, we obtain the entries of the matrix $A_{n-1}$ :

$$
\begin{aligned}
& l_{j}^{\prime \prime}\left(x_{i}\right)=-(-1)^{i+j} \frac{2-x_{i} x_{j}-x_{i}^{2}}{\left(x_{i}-x_{j}\right)^{2}\left(1-x_{i}^{2}\right)}, \quad i \neq j, \quad i=1, \ldots, n-1, \\
& l_{i}^{\prime \prime}\left(x_{i}\right)=\frac{-1}{3\left(1-x_{i}^{2}\right)}\left(n^{2}+2+\frac{3 x_{i}^{2}}{1-x_{i}^{2}}\right), \quad i=1, \ldots, n-1 .
\end{aligned}
$$

Evaluating instead at the new nodes $\mathbf{z}$ yields the entries of the matrix $B_{n-1}$ :

$$
\begin{aligned}
l_{j}^{\prime \prime}\left(z_{i}\right) & =\frac{-2(-1)^{n+j}}{n^{2}}\left(\frac{n^{2} T_{n}\left(z_{i}\right)\left(z_{i}-x_{j}\right)+\left(1-z_{i} x_{j}\right) T_{n}^{\prime}\left(z_{i}\right)}{\left(z_{i}-x_{j}\right)^{3}}\right) \\
& =\frac{-2(-1)^{j}}{n}\left(\frac{n\left(z_{i}-x_{j}\right) \cos n \zeta_{i}-\left(1-z_{i} x_{j}\right) T_{n}^{\prime}\left(z_{i}\right)}{\left(z_{i}-x_{j}\right)^{3}}\right), \quad \text { with } z_{i}=-\cos \zeta_{i} \quad i=1, \ldots, n-1,
\end{aligned}
$$

except for $n$ even and $i=j=n / 2$, where $z_{(n / 2)}=x_{(n / 2)}=0$ and

$$
l_{n / 2}^{\prime \prime}(0)=-\frac{n^{2}+2}{3}
$$

Let now $M_{n-1}$ be the matrix of dimension $n-1$ given by (13), where $\mathbf{x}$ is the Chebyshev grid. Experimentally, we can check that the eigenvalues of both the two matrices $M_{n-1}^{-1} A_{n-1}$ and $M_{n-1}^{-1} B_{n-1}$ are real, positive and very well-behaved. Let $\lambda_{n, m}^{A}$ and $\lambda_{n, m}^{B}, m=1, \ldots, n-1$, be the eigenvalues, ordered increasingly, of $M_{n-1}^{-1} A_{n-1}$ and $M_{n-1}^{-1} B_{n-1}$ respectively. In [11], the following explicit expression for the Chebyshev preconditioned eigenvalues $\lambda_{n, m}^{A}, m=1, \ldots, n-1$, is given:

$$
\lambda_{n, m}^{A}=n(n+1) \frac{\sin ^{2}\left(\frac{\pi}{2 n}\right) \cos \left(\frac{\pi}{2 n}\right)}{\sin \left(\frac{m \pi}{2 n}\right) \sin \frac{(m+1) \pi}{2 n}}, \quad m=1, \ldots, n-1 .
$$

Consequently, the following estimate holds:

$$
1 \leq \lambda_{n, m}^{A}<\pi^{2} / 4, \quad m=1, \ldots, n-1 .
$$


TABLE 2. Maximum preconditioned eigenvalues for the standard Chebyshev collocation method $\left(\lambda_{n, n-1}^{A}\right)$, compared to those of the superconsistent Chebyshev collocation method $\left(\lambda_{n, n-1}^{B}\right)$.

\begin{tabular}{ccc|ccc}
\hline$n$ & $\lambda_{n, n-1}^{A}$ & $\lambda_{n, n-1}^{B}$ & $n$ & $\lambda_{n, n-1}^{A}$ & $\lambda_{n, n-1}^{B}$ \\
\hline 5 & 1.90 & 1.72 & 13 & 2.26 & 2.13 \\
6 & 2.00 & 1.82 & 14 & 2.28 & 2.15 \\
7 & 2.07 & 1.89 & 15 & 2.29 & 2.17 \\
8 & 2.13 & 1.95 & 16 & 2.30 & 2.18 \\
9 & 2.17 & 2.00 & 17 & 2.31 & 2.20 \\
10 & 2.20 & 2.04 & 18 & 2.32 & 2.21 \\
11 & 2.22 & 2.07 & 19 & 2.33 & 2.22 \\
12 & 2.24 & 2.10 & 20 & 2.33 & 2.24 \\
\hline
\end{tabular}

For the superconsistent preconditioned eigenvalues $\lambda_{n, m}^{B}, m=1, \ldots, n-1$, we did not find any explicit expression. However, numerical experiments show an even better result, that is:

$$
1 \leq \lambda_{n, m}^{B}<\lambda_{n, m}^{A}, \quad m=1, \ldots, n-1
$$

In Table 2 we give the values of the maximum preconditioned eigenvalues $\lambda_{n, n-1}^{A}$ and $\lambda_{n, n-1}^{B}$ for various $n$. We note that $\lambda_{n, 1}^{A}=1$ and $\lambda_{n, 1}^{B}=\lambda_{n, 2}^{B}=1$. It is also worthwhile to note that the matrix $M_{n-1}$ does not depend on the grid $\mathbf{z}$.

\section{Convergence analysis for the superconsistent Chebyshev method}

The convergence, for $n \rightarrow+\infty$, of the discrete solution $u_{n}$, obtained by collocation at the Chebyshev nodes, to the exact solution $u$ of problem (5) is proved in [2], where estimates showing the spectral decay of the error are given. Before briefly discussing some convergence results of the superconsistent discrete solution $w_{n}$ to $u$ for $n \rightarrow+\infty$ (we refer to [4] for a complete and detailed convergence analysis), we introduce here some interesting lemmas which play an important role in the proof of convergence. In particular a suitable quadrature formula is going to be introduced and studied.

We will denote by $\mathbb{P}_{n}$ the space of polynomials of degree less than or equal to $n$.

Lemma 3.1. Let $\chi_{n+1}=\left(1-x^{2}\right) T_{n}^{\prime}$. Then, the following orthogonality relation holds:

$$
\int_{-1}^{1} \chi_{n+1}^{\prime \prime} q_{n} \omega \mathrm{d} x=0, \text { with } \omega(x)=\frac{1}{\sqrt{1-x^{2}}}
$$

for all $q_{n}$ polynomials of degree $n$ with $q_{n}( \pm 1)=0$ and $q_{n}^{\prime}( \pm 1)=0$.

Proof. Let $q_{n}$ be a polynomial of degree $n$ with $q_{n}( \pm 1)=0$ and $q_{n}^{\prime}( \pm 1)=0$. Then, we can write

$$
q_{n}(x)=\left(1-x^{2}\right) s_{n-2}(x)
$$

where $s_{n-2}$ is a polynomial of degree $n-2$ with $s_{n-2}( \pm 1)=0$. Integrating by parts twice and using the boundary constraints, we have:

$$
\int_{-1}^{1} \chi_{n+1}^{\prime \prime} q_{n} \omega \mathrm{d} x=\int_{-1}^{1} \chi_{n+1}^{\prime \prime}\left(1-x^{2}\right) s_{n-2} \omega \mathrm{d} x=\int_{-1}^{1} \chi_{n+1}\left[\left(1-x^{2}\right) s_{n-2} \omega\right]^{\prime \prime} \mathrm{d} x .
$$


Now, we recall that the derivatives of Chebyshev polynomials form a set of orthogonal functions (see, for instance, [19]), i.e.:

$$
\int_{-1}^{1} T_{n}^{\prime} T_{m}^{\prime}\left(1-x^{2}\right) \omega \mathrm{d} x=0, \quad \forall n \neq m
$$

Thus, we have:

$$
\int_{-1}^{1} T_{n}^{\prime} r_{n-1}^{\prime}\left(1-x^{2}\right) \omega \mathrm{d} x=0, \quad \forall r_{n-1} \in \mathbb{P}_{n-1}
$$

Recalling the definition of $\chi_{n+1}$, we can write:

$$
\int_{-1}^{1} \chi_{n+1}\left[\left(1-x^{2}\right) s_{n-2} \omega\right]^{\prime \prime} \mathrm{d} x=\int_{-1}^{1} T_{n}^{\prime} \frac{\left[\left(1-x^{2}\right) s_{n-2} \omega\right]^{\prime \prime}}{\omega}\left(1-x^{2}\right) \omega \mathrm{d} x=0 .
$$

The last equality in (20) is true because $\left[\left(1-x^{2}\right) s_{n-2} \omega\right]^{\prime \prime} / \omega$ is a polynomial of degree less then or equal to $n-1$, under the hypothesis $s_{n-2}( \pm 1)=0$. Combining with $(19)$ this concludes the proof.

This lemma provides a link between the Chebyshev superconsistent grid and the so called Sobolev-type orthogonal polynomials (see $[13,16]$ ). In fact, given a function $f$, the set of nodes $\mathbf{z}$ is related to a Gauss integration formula of the type:

$$
\int_{-1}^{1} f \omega \mathrm{d} x \approx \sum_{i=0}^{n} f\left(z_{i}\right) \omega_{i}+f^{\prime}(-1) \tilde{\omega}_{0}+f^{\prime}(1) \tilde{\omega}_{n}
$$

where $z_{0}=-1, z_{n}=1$ and $\tilde{\omega}_{0}, \tilde{\omega}_{n}$ and $\omega_{i}, i=0, \ldots, n$, are suitable weights. As a matter of fact, we can prove the following results.

Lemma 3.2. The quadrature formula (21) is exact if $f$ is a polynomial of degree less than or equal to $2 n-1$.

Proof. Let us choose a Lagrange type polynomial basis as follows:

$$
\begin{gathered}
\hat{l}_{i} \in \mathbb{P}_{n+2}, i=0, \ldots, n, \text { such that: } \hat{l}_{i}\left(z_{j}\right)=\delta_{i j}, \hat{l}_{i}^{\prime}( \pm 1)=0, j=1, \ldots, n-1, \\
\tilde{l}_{0} \in \mathbb{P}_{n+2} \text { such that: } \tilde{l}_{0}\left(z_{i}\right)=0, \quad i=0, \ldots, n, \quad \tilde{l}_{0}^{\prime}(-1)=1, \quad \tilde{l}_{0}^{\prime}(1)=0, \\
\tilde{l}_{n} \in \mathbb{P}_{n+2} \text { such that: } \tilde{l}_{n}\left(z_{i}\right)=0, \quad i=0, \ldots, n, \quad \tilde{l}_{n}^{\prime}(-1)=0, \tilde{l}_{n}^{\prime}(1)=1 .
\end{gathered}
$$

Afterwards, we define:

$$
\omega_{i}=\int_{-1}^{1} \hat{l}_{i} \omega \mathrm{d} x, \quad i=0, \ldots, n, \quad \tilde{\omega}_{0}=\int_{-1}^{1} \tilde{l}_{0} \omega \mathrm{d} x, \quad \tilde{\omega}_{n}=\int_{-1}^{1} \tilde{l}_{n} \omega \mathrm{d} x
$$

With this setting it should be clear that formula $(21)$ is exact for any polynomial $f$ of degree $n+2$. Let us also note that $\tilde{\omega}_{0}=-\tilde{\omega}_{n}$. To prove the exactness up to the degree $2 n-1$, let us consider the two following interpolation operators. Let $\hat{I}_{n+2} f$ be the interpolant of $f$ constructed with the help of the Lagrange basis introduced above, i.e.:

$$
\left(\hat{I}_{n+2} f\right)\left(z_{i}\right)=f\left(z_{i}\right), \quad i=0, \ldots, n, \quad\left(\hat{I}_{n+2} f\right)^{\prime}( \pm 1)=f^{\prime}( \pm 1)
$$

Let $I_{n-2}^{\{\mathbf{z}\}} f$ be the interpolant of $f$ at the nodes $\mathbf{z}$, i.e.:

$$
\left(I_{n-2}^{\{\mathbf{z}\}} f\right)\left(z_{i}\right)=f\left(z_{i}\right), \quad i=1, \ldots, n-1 .
$$


First of all, let us prove that the quadrature formula (21) is exact for any polynomial $f$ of degree $2 n-1$ satisfying $f( \pm 1)=0$ and $f^{\prime}( \pm 1)=0$. Actually, in this case we can write $f=\left(1-x^{2}\right)^{2} g$ with $g \in \mathbb{P}_{2 n-5}$. Since:

$$
\hat{I}_{n+2} f=\left(1-x^{2}\right)^{2} I_{n-2}^{\{\mathbf{z}\}} g,
$$

we have:

$$
f-\hat{I}_{n+2} f=\left(1-x^{2}\right)^{2}\left[g-I_{n-2}^{\{\mathbf{z}\}} g\right]=\left(1-x^{2}\right)^{2} \chi_{n+1}^{\prime \prime} h, \quad \text { with } \quad h \in \mathbb{P}_{n-4} .
$$

Lemma 3.1 implies that:

$$
\int_{-1}^{1}\left(1-x^{2}\right)^{2} \chi_{n+1}^{\prime \prime} h \omega \mathrm{d} x=0
$$

hence

$$
\int_{-1}^{1} f \omega \mathrm{d} x=\int_{-1}^{1} \hat{I}_{n+2} f \omega \mathrm{d} x=\sum_{i=0}^{n} f\left(z_{i}\right) \omega_{i}+f^{\prime}(-1) \tilde{\omega}_{0}+f^{\prime}(1) \tilde{\omega}_{n}=\sum_{i=1}^{n-1} f\left(z_{i}\right) \omega_{i},
$$

where we used that the formula is exact up to degree $n+2$. This shows that the quadrature formula $(21)$ is exact for any $f \in \mathbb{P}_{2 n-1}$ with $f( \pm 1)=0$ and $f^{\prime}( \pm 1)=0$.

Now, for an arbitrary $f \in \mathbb{P}_{2 n-1}$, we can write

$$
f=\bar{f}+r_{3}, \quad \bar{f} \in \mathbb{P}_{2 n-1}, \quad r_{3} \in \mathbb{P}_{3},
$$

with

and

$$
\bar{f}( \pm 1)=0, \quad \bar{f}^{\prime}( \pm 1)=0,
$$

In this case we obtain:

$$
r_{3}(-1)=f(-1), r_{3}(1)=f(1), r_{3}^{\prime}(-1)=f^{\prime}(-1), r_{3}^{\prime}(1)=f^{\prime}(1) .
$$

$$
\begin{aligned}
\int_{-1}^{1} f \omega \mathrm{d} x= & \int_{-1}^{1} \bar{f} \omega \mathrm{d} x+\int_{-1}^{1} r_{3} \omega \mathrm{d} x=\sum_{i=0}^{n} \bar{f}\left(z_{i}\right) \omega_{i}+\bar{f}^{\prime}(-1) \tilde{\omega}_{0}+\bar{f}^{\prime}(1) \tilde{\omega}_{n} \\
& +\sum_{i=0}^{n} r_{3}\left(z_{i}\right) \omega_{i}+r_{3}^{\prime}(-1) \tilde{\omega}_{0}+r_{3}^{\prime}(1) \tilde{\omega}_{n}=\sum_{i=0}^{n} f\left(z_{i}\right) \omega_{i}+f^{\prime}(-1) \tilde{\omega}_{0}+f^{\prime}(1) \tilde{\omega}_{n}
\end{aligned}
$$

so that the quadrature formula (21) is exact for any polynomial $f \in \mathbb{P}_{2 n-1}$.

The theoretical proof of the convergence, for $n \rightarrow+\infty$, of the discrete solution $w_{n}$ of (7) (obtained by collocation at the superconsistent Chebyshev grid) to the solution $u$ of (5) is basically obtained from the properties of the quadrature formula (21). More specifically the knowledge of the behavior of nodes and weights of that formula, and in particular the fact that the weights in (21) are positive (except one), are of crucial relevance for the development of a convergence theory. The proof of these important facts is actually given in [4]. We review here the main steps of the convergence proof. For a more rigorous analysis we refer to Theorem 9 in [4].

Let us begin by observing that from (7) we can write:

$$
\left.-w_{n}^{\prime \prime}=I_{n-2}^{\{\mathbf{z}\}} f \text { in }\right]-1,1[
$$

since $w_{n}^{\prime \prime}$ and $I_{n-2}^{\{\mathbf{z}\}}$ have both degree $n-2$ and coincide in $n-1$ points.

On the other hand, denoting by $I_{n-2}^{\{\mathbf{x}\}} f$ the interpolant of $f$ at the nodes $\mathbf{x}$, i.e.:

$$
\left(I_{n-2}^{\{\mathbf{x}\}} f\right)\left(x_{i}\right)=f\left(x_{i}\right), \quad i=1, \ldots, n-1,
$$


we have that

$$
\left.-u_{n}^{\prime \prime}=I_{n-2}^{\{\mathbf{x}\}} f \quad \text { in }\right]-1,1[.
$$

After introducing the weighted Sobolev spaces $\mathrm{L}_{\omega}^{2}=\mathrm{L}_{\omega}^{2}(-1,1)$ and $\mathrm{H}_{0, \omega}^{1}=\mathrm{H}_{0, \omega}^{1}(-1,1)$ in the usual way, by the triangle inequality, we have:

$$
\left\|u-w_{n}\right\|_{\mathrm{H}_{0, \omega}^{1}} \leq\left\|u-u_{n}\right\|_{\mathrm{H}_{0, \omega}^{1}}+\left\|u_{n}-w_{n}\right\|_{\mathrm{H}_{0, \omega}^{1}} .
$$

We already know that the first term on the right-hand side of (24) converges to zero, so that we now estimate the second one. From (22) and (23) we get:

$$
\left.-\left(u_{n}-w_{n}\right)^{\prime \prime}=I_{n-2}^{\{\mathbf{x}\}} f-I_{n-2}^{\{\mathbf{z}\}} f \quad \text { in }\right]-1,1[.
$$

By an inequality proven in [2] (see also [5], p. 192), equation (25) implies:

$$
\begin{aligned}
\left\|u_{n}-w_{n}\right\|_{\mathrm{H}_{0, \omega}^{1}}^{2} & \leq C_{1} \int_{-1}^{1}\left(u_{n}-w_{n}\right)^{\prime}\left(\left(u_{n}-w_{n}\right) \omega\right)^{\prime} \mathrm{d} x \\
& =-C_{1} \int_{-1}^{1}\left(u_{n}-w_{n}\right)^{\prime \prime}\left(u_{n}-w_{n}\right) \omega \mathrm{d} x=C_{1} \int_{-1}^{1}\left(I_{n-2}^{\{\mathbf{x}\}} f-I_{n-2}^{\{\mathbf{z}\}} f\right)\left(u_{n}-w_{n}\right) \omega \mathrm{d} x,
\end{aligned}
$$

where $C_{1}$ is a constant not depending on $n$.

In [4], the following inequality has been proven:

$$
\left\|u_{n}-w_{n}\right\|_{\mathrm{H}_{0, \omega}^{1}} \leq C_{2} \frac{1}{\sqrt{n}}\left\|f-I_{n-2}^{\{\mathbf{x}\}} f\right\|_{\mathrm{L}^{\infty}}
$$

for some constant $C_{2}$ not depending on $n$. The above inequality, that strongly uses the properties of the quadrature formula (21), is crucial to end the convergence proof, since from (24) and (26) we obtain:

$$
\left\|u-w_{n}\right\|_{\mathrm{H}_{0, \omega}^{1}} \leq\left\|u-u_{n}\right\|_{\mathrm{H}_{0, \omega}^{1}}+C_{3} \frac{1}{\sqrt{n}}\left\|f-I_{n-2}^{\{\mathbf{x}\}} f\right\|_{\mathrm{L}^{\infty}},
$$

for some positive constant $C_{3}$ not depending on $n$.

In conclusion, the spectral convergence, for $n \rightarrow+\infty$, of $w_{n}$ to the exact solution $u$ comes from the convergence, for $n \rightarrow+\infty$, of $u_{n}$ to $u$ and of $I_{n-2}^{\{\mathbf{x}\}} f$ to $f$. This is a well-established result (see, for example, [3]).

Let us remark that the error estimate (27) is not optimal, due to the term $n^{-1 / 2}$ appearing on the right-hand side of that inequality. Nevertheless, numerical experiments, part of them reported in the coming two sections, show that the error converges to zero more rapidly than estimated.

\section{LebesGue constants}

In this section we present some numerical results about the Lebesgue constant associated with the distributions of nodes examined in the previous sections.

We remind that the Lebesgue constant associated with a generic distribution of $n+1$ nodes $x_{j}, j=0, \ldots, n$ in $[-1,1]$, with $x_{0}=-1$ and $x_{n}=1$, is defined as:

$$
\Lambda_{n}=\max _{x \in[-1,1]} \sum_{j=0}^{n}\left|l_{j}(x)\right|,
$$



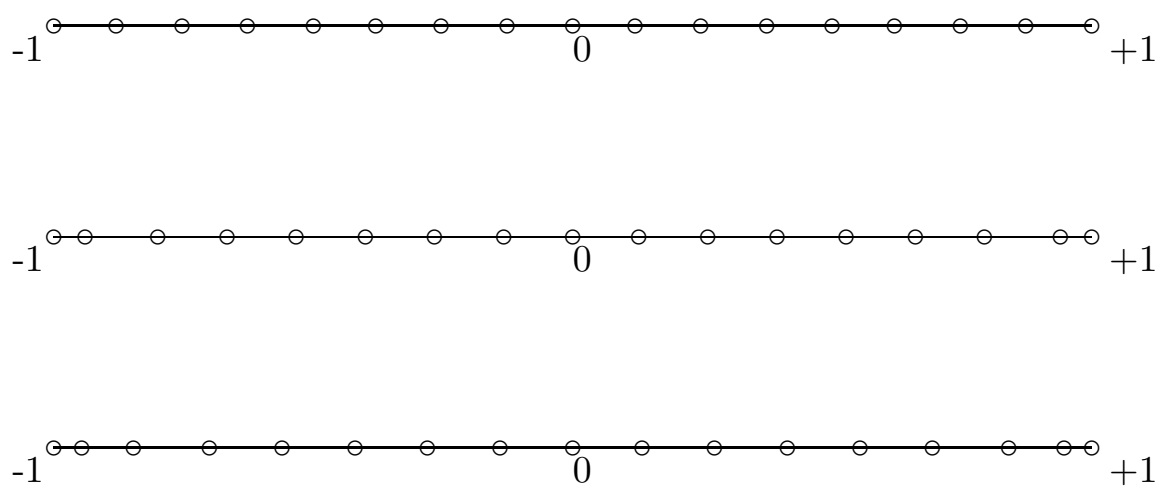

Figure 2. Initial (equispaced) distribution and the first two iterations of the recursive algorithm for $n=16$.

where $\left\{l_{j}\right\}_{j=0, \ldots, n}$ is the Lagrange basis with respect to the set of nodes. An equivalent definition is:

$$
\Lambda_{n}=\sup _{f \in C^{0}([-1,1]), f \neq 0} \frac{\left\|I_{n} f\right\|_{\mathrm{L}^{\infty}(-1,1)}}{\|f\|_{\mathrm{L}^{\infty}(-1,1)}}
$$

where $I_{n}: C^{0}([-1,1]) \rightarrow \mathbb{P}_{n}$ is the interpolation operator at the nodes.

It is known (see [12]) that, for any given $n \geq 2$, there exists a unique distribution of nodes minimizing the Lebesgue constant, although its computation turns out to be quite a hard optimization problem. Both the distribution of the Legendre Gauss-Lobatto nodes (zeros of $\left(1-x^{2}\right) P_{n}^{\prime}$ ) and the one of the Chebyshev GaussLobatto nodes (zeros of $\left(1-x^{2}\right) T_{n}^{\prime}$ ) are very close to minimize $\Lambda_{n}$. Another very good distribution (see [12]), probably the best from a practical point of view, is the one obtained by taking the zeros of $T_{n+1}$ and applying a dilation in order to fit the first and the last of these points to the endpoints \pm 1 . This is achieved by dividing the zeros of $T_{n+1}$ by $\cos (\pi / 2(n+1))$. Equispaced nodes give instead very high values of the Lebesgue constant.

In Table 3 we show the values of the Lebesgue constant for different distributions of nodes. We note that the set of superconsistent Chebyshev nodes $z_{i}, i=1, \ldots, n-1$, introduced in Section 2, with the addition of the two points $z_{0}=-1$ and $z_{n}=1$, has a Lebesgue constant which is less than the one associated with the distribution of the Chebyshev Gauss-Lobatto nodes.

In [9] the following iterative procedure was suggested. Given an initial distribution $\mathbf{x}$ of $n-1$ points in ]-1, 1[, we proceed according to the following:

\section{Recursive algorithm}

1. Construct $\chi_{n+1}$ to be the polynomial of degree $n+1$, vanishing at $\mathbf{x} \cup\{ \pm 1\}$;

2. Compute the grid $\mathbf{z}$ given by the $n-1$ zeros of $\chi_{n+1}^{\prime \prime}$;

3. Replace the grid $\mathbf{x}$ by $\mathbf{z}$;

4. Go to 1 .

We noticed in Section 1 (Thm. 1.1) that we expect to have $\mathbf{x}=\mathbf{z}$ only when $\mathbf{x}$ is the set of the zeros of the derivative of the Legendre polynomial of degree $n$. Therefore, if we start from this set, the sequence turns out to be constant. Thus, the Gauss-Lobatto Legendre distribution is a stable fixed-point and the unique limit of the sequence constructed using the recursive algorithm. 
TABLE 3. Lebesgue constant for different distribution of nodes: (1) equispaced; (2) Chebyshev Gauss-Lobatto: zeros of $T_{n}^{\prime} \cup\{ \pm 1\}$; (3) superconsistent Chebyshev: zeros of $\chi_{n+1}^{\prime \prime} \cup\{ \pm 1\}$ with $\chi_{n+1}=\left(1-x^{2}\right) T_{n}^{\prime}$; (4) Legendre Gauss-Lobatto: zeros of $P_{n}^{\prime} \cup\{ \pm 1\}$; (5) dilated Gauss: zeros of $T_{n+1}$ divided by $\cos (\pi / 2(n+1))$; (6) optimal distribution.

\begin{tabular}{ccccccc}
\hline$n$ & $(1)$ & $(2)$ & $(3)$ & $(4)$ & $(5)$ & $(6)$ \\
\hline 7 & 6.929 & 2.202 & 2.107 & 1.972 & 1.866 & 1.852 \\
8 & 10.945 & 2.274 & 2.188 & 2.045 & 1.941 & 1.925 \\
9 & 17.848 & 2.361 & 2.282 & 2.120 & 2.008 & 1.996 \\
10 & 29.899 & 2.420 & 2.348 & 2.180 & 2.068 & 2.053 \\
11 & 51.214 & 2.489 & 2.422 & 2.241 & 2.123 & 2.129 \\
12 & 89.324 & 2.539 & 2.476 & 2.291 & 2.174 & 2.170 \\
13 & 158.102 & 2.595 & 2.537 & 2.342 & 2.221 & 2.205 \\
14 & 283.211 & 2.638 & 2.583 & 2.386 & 2.265 & 2.248 \\
15 & 512.351 & 2.686 & 2.634 & 2.430 & 2.306 & 2.291 \\
16 & 934.533 & 2.724 & 2.675 & 2.468 & 2.344 & 2.328 \\
17 & 1716.460 & 2.766 & 2.719 & 2.507 & 2.381 & 2.364 \\
18 & 3171.365 & 2.800 & 2.756 & 2.541 & 2.415 & 2.398 \\
19 & 5889.585 & 2.837 & 2.795 & 2.575 & 2.448 & 2.429 \\
20 & 10986.700 & 2.867 & 2.827 & 2.606 & 2.479 & 2.463 \\
\hline
\end{tabular}

TABLE 4. Lebesgue constants, for $n=16$, relative to the distributions of points in $[-1,1]$, obtained by applying 19 iterations of the recursive algorithm of Section 4 . The first value (iteration 0) refers to the Lebesgue constant of the equispaced distribution.

\begin{tabular}{cccc}
\hline Iteration & Lebesgue constant & Iteration & Lebesgue constant \\
\hline 0 & 934.53 & 10 & 2.48 \\
1 & 126.79 & 11 & 2.43 \\
2 & 29.90 & 12 & 2.40 \\
3 & 11.71 & 13 & 2.39 \\
4 & 6.40 & 14 & 2.39 \\
5 & 4.34 & 15 & 2.39 \\
6 & 3.39 & 16 & 2.41 \\
7 & 2.97 & 17 & 2.42 \\
8 & 2.72 & 18 & 2.43 \\
9 & 2.57 & 19 & 2.44 \\
\hline
\end{tabular}

It is interesting to observe that such a limit distribution has quite a large basin of attraction, since the procedure converges even if the starting grid is equispaced. In Figure 2, for $n=16$, we compare the grids corresponding to the initial equispaced distribution and two subsequent iterations. The last of them is already very similar to a Gauss type distribution of nodes. The convergence is fast as we can check from Table 4, where we give the Lebesgue constants relative to the sequence of grids obtained starting from the equispaced grid. These numbers are rapidly approaching the value 2.468 (see Tab. 3, column (4) for $n=16$ ). Note also that, during the iteration process, there are distributions with a Lebesgue constant lower than the limit value, remaining, of course, above the minimum which is 2.328 (see Tab. 3, column (6) for $n=16$ ). Similar results hold for different values of $n$. 


\section{Superconsistent discretizations of the AdVECTiOn-Diffusion operator}

Let now $L$ in (1) be the advection-diffusion operator: i.e. $L=-\epsilon \frac{\mathrm{d}^{2}}{\mathrm{~d} x^{2}}+\beta \frac{\mathrm{d}}{\mathrm{d} x}$, where $\epsilon>0, \beta$ are two constants. Therefore, for a given function $f$, we will consider the Dirichlet boundary-value problem:

$$
\left.-\epsilon u^{\prime \prime}+\beta u^{\prime}=f \quad \text { in }\right]-1,1[, \quad \text { with } \quad u(-1)=u(1)=0 .
$$

Based on the same ideas developed in the previous sections, we can find the approximated solution of (28) following two different strategies. The classical one consists in finding a discrete function $u_{n} \in \mathbb{P}_{n}^{0}$, solution of the following standard collocation problem:

$$
\left[-\epsilon u_{n}^{\prime \prime}+\beta u_{n}^{\prime}\right]\left(x_{i}\right)=f\left(x_{i}\right) \quad \text { for } \quad i=1, \ldots, n-1
$$

where the nodes $x_{i}, i=1, \ldots, n-1$, can be either the Legendre or the Chebyshev nodes, that is the zeros of $P_{n}^{\prime}$ or $T_{n}^{\prime}$ respectively.

The second strategy introduces a special superconsistent grid $\mathbf{z}$ and requires the determination of a new solution $w_{n} \in \mathbb{P}_{n}^{0}$ such that:

$$
\left[-\epsilon w_{n}^{\prime \prime}+\beta w_{n}^{\prime}\right]\left(z_{i}\right)=f\left(z_{i}\right) \quad \text { for } \quad i=1, \ldots, n-1
$$

Arguing as in (9), the collocation grid $\mathbf{z}$ is determined by solving the equation:

$$
\left(L \chi_{n+1}\right)\left(z_{i}\right)=-\epsilon \chi_{n+1}^{\prime \prime}\left(z_{i}\right)+\beta \chi_{n+1}^{\prime}\left(z_{i}\right)=0, \quad i=1, \ldots, n-1,
$$

where $\chi_{n+1}=\left(1-x^{2}\right) P_{n}^{\prime}$, if we look for a superconsistent Legendre grid, or $\chi_{n+1}=\left(1-x^{2}\right) T_{n}^{\prime}$ if we are interested in working with a superconsistent Chebyshev grid.

In particular, for the superconsistent Legendre grid, thanks to (10), we have:

$$
\left(L \chi_{n+1}\right)\left(z_{i}\right)=n(n+1)\left[\epsilon P_{n}^{\prime}\left(z_{i}\right)-\beta P_{n}\right]\left(z_{i}\right)=0, \quad i=1, \ldots, n-1
$$

The above equation has actually $n$ distinct zeros, but one of them is not interesting to us since, in general, is outside the interval $[-1,1]$ (it goes to infinity for $\beta$ tending to zero). We address to [6] for more information and other conjectures about the exact localization of these nodes. In short, each $z_{i}$ is positioned upwind with respect to the corresponding point $x_{i}$. We obtain $z_{i}=x_{i}, i=1, \ldots, n-1$, if and only if $\beta=0$.

In the Chebyshev case, by writing (14) and (15) in trigonometric way, it is easy to see that:

$$
-\epsilon \chi_{n+1}^{\prime \prime}\left(z_{i}\right)+\beta \chi_{n+1}^{\prime}\left(z_{i}\right)=0 \Leftrightarrow F(\xi)=0, \quad i=1, \ldots, n-1,
$$

where the function $F$ is defined as follows:

$$
\begin{aligned}
F(\xi)=-\epsilon\left(n^{2}+1\right) n \sin (n \xi) \sin ^{2} \xi+\epsilon \xi n & \cos \xi \sin (n \xi) \\
& -\epsilon \xi n^{2} \sin \xi \cos (n \xi)-\beta n^{2} \cos (n \xi) \sin ^{3} \xi-\beta n \cos \xi \sin (n \xi) \sin ^{2} \xi
\end{aligned}
$$

Also this equation has $n$ zeros. By numerical evidence it turns out that $n-1$ of these zeros belong to ]- $1,1[$ for any choice of the coefficients $\beta$ and $\epsilon$. We eliminate the unique zero that tends to infinity when $\beta$ goes to zero. This choice is motivated by the fact that, when $\beta=0$, we must reobtain the zeros given by (16).

To find the zeros of $F$ defined in (32) it is helpful to have some more information about their location. Let us suppose for instance that $\beta>0$, and let us denote by $\left[a_{j}, b_{j}\right], j=1, \ldots, n-1$, the $n-1$ intervals defined by:

$$
a_{j}=\frac{2 j-1}{2 n} \pi, \quad b_{j}=\frac{2 j+1}{2 n} \pi, \quad j=1, \ldots, n-2,
$$



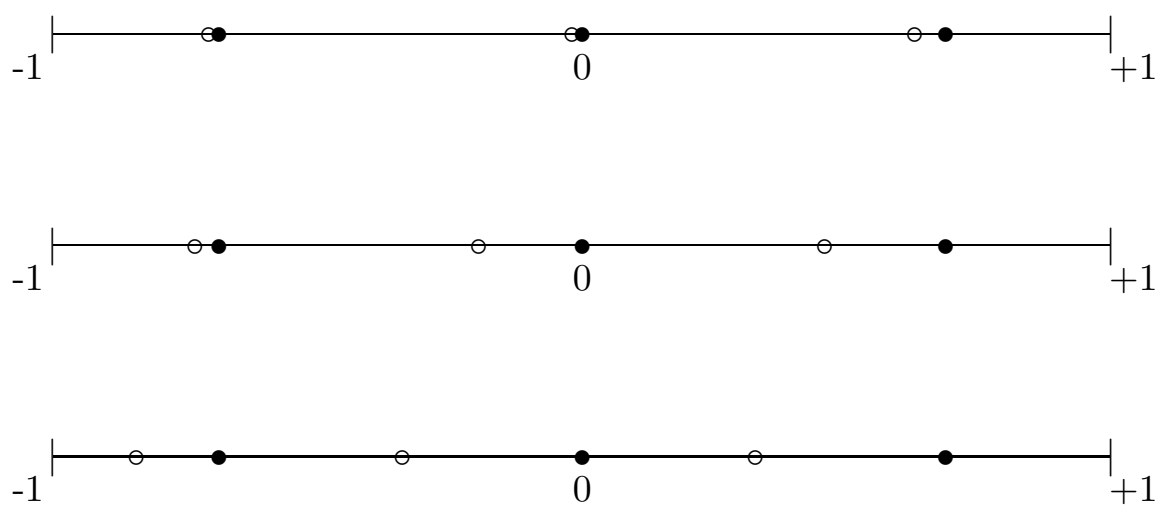

Figure 3. The grid $\mathbf{x}$ (black circles) and the grid $\mathbf{z}$ (white circles) for the superconsistent Chebyshev method with $n=4, \beta=1$ and three different choices of the diffusion parameter, namely: $\epsilon=1, \epsilon=0.1$ and $\epsilon=0.01$.

and

$$
a_{n-1}=\frac{n-2}{n} \pi, \quad b_{n-1}=\frac{n-1}{n} \pi .
$$

Numerical experiments bring to the conclusion that each interval contains a unique zero of $F$. From these estimates we can deduce that each $z_{i}$ is located upwind with respect the corresponding point $x_{i}$. Figure 3 shows, for $n=4$ and $\beta=1$, the distribution of the nodes $z_{i}, i=1, \ldots, 3$ (white circles) and the one of the nodes $x_{i}, i=1, \ldots, 3$ (black circles) for $\epsilon=1, \epsilon=0.1$ and $\epsilon=0.01$. Let us note again that, in the Chebyshev case, when $\beta=0$, we do not have the equivalence $z_{i}=x_{i}, i=1, \ldots, n-1$ as in the Legendre case.

It is not difficult to write the linear systems associated to (29) and (30) by differentiating the Lagrange polynomials (see (12) and (18)) and evaluating at the collocation nodes. The discretization matrices associated to the linear systems arising from (29) and (30) are given respectively by:

$$
A_{n-1}=\left\{-\epsilon l_{j}^{\prime \prime}\left(x_{i}\right)+\beta l_{j}^{\prime}\left(x_{i}\right)\right\}_{i, j=1, \ldots, n-1},
$$

and

$$
B_{n-1}=\left\{-\epsilon l_{j}^{\prime \prime}\left(z_{i}\right)+\beta l_{j}^{\prime}\left(z_{i}\right)\right\}_{i, j=1, \ldots, n-1} .
$$

For the advection-diffusion problem the use of the superconsistent method (both in the Legendre and the Chebyshev case) brings to a substantial improvement when compared to the standard collocation method. For the one-dimensional Legendre case, some numerical tests were given in [6], and, for two-dimensional problems, in [7]. Here we show some tests concerning the Chebyshev case.

It is known that the standard collocation method is not stabilized with respect to the parameter $\epsilon$, by meaning that wide oscillations are observed when $n$ is not sufficiently large and $\epsilon$ is small compared to $|\beta|$. In Figure 4 we show, for example, the exact solution of problem (28) when

$$
f=1, \quad \epsilon=0.001, \quad \beta=1 .
$$




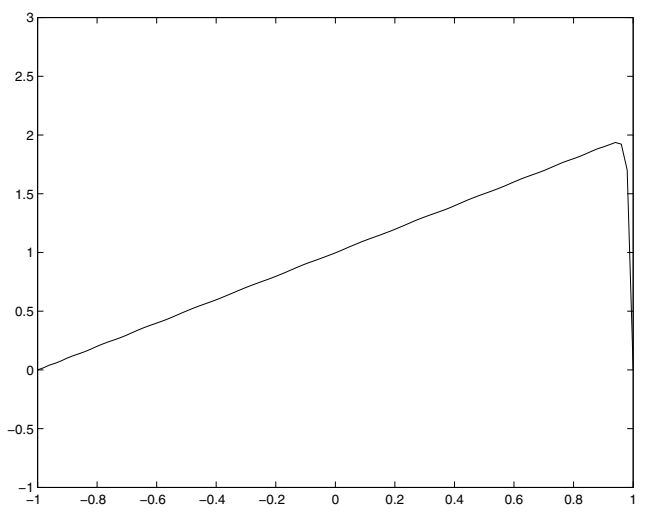

FigURE 4. Exact solution of problem (28) with $f=1, \epsilon=0.001$ and $\beta=1$.
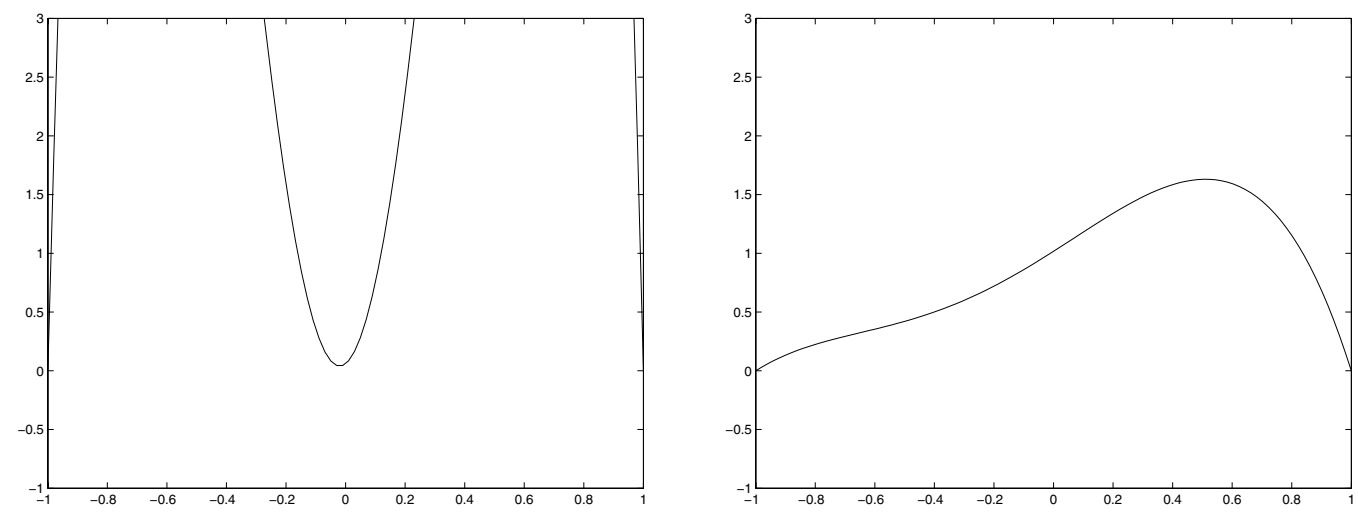

Figure 5. Approximated solution $u_{n}$ (left) and $w_{n}$ (right) of problem (28) with $f=1, \epsilon=$ $0.001, \beta=1$ and $n=4$ obtained with standard collocation (left) and superconsistent collocation (right) respectively.

The behavior of the approximated solution $u_{n}$ obtained by collocating at the standard Chebyshev nodes $x_{i}$, $i=1, \ldots, n-1$, is shown in Figure 5 for $n=4$ and Figure 6 for $n=11$. As expected, the boundary layer at the point $x=1$ gives rise to inaccurate solutions. Moreover, $u_{n}$ blows up for $n$ fixed and $\epsilon$ tending to zero, which means that there is no stabilization with respect to $\epsilon$. The situation definitely changes after collocating at the superconsistent Chebyshev nodes $z_{i}, i=1, \ldots, n-1$ (see Figs. 5 and 6 ). As the reader can see, now the approximated solution $w_{n}$ is bounded independently of $\epsilon$. More accuracy can be only obtained by increasing $n$. We should note, in fact, that, for $\epsilon$ fixed, both $u_{n}$ and $w_{n}$ converge to $u$ exponentially. The superconsistent approach is therefore suggested when, for practical reasons, $n$ cannot be larger than a certain limit.

We conclude this section showing that also in the case of the advection-diffusion operator the use of superconsistent grids allows the construction of very-effective finite-difference preconditioners.

Let us consider the matrix $M_{n-1}$, of dimension $n-1$, related to the finite-difference discretization of the advection-diffusion operator $L=-\epsilon \frac{\mathrm{d}^{2}}{\mathrm{~d} x^{2}}+\beta \frac{\mathrm{d}}{\mathrm{d} x}$. 

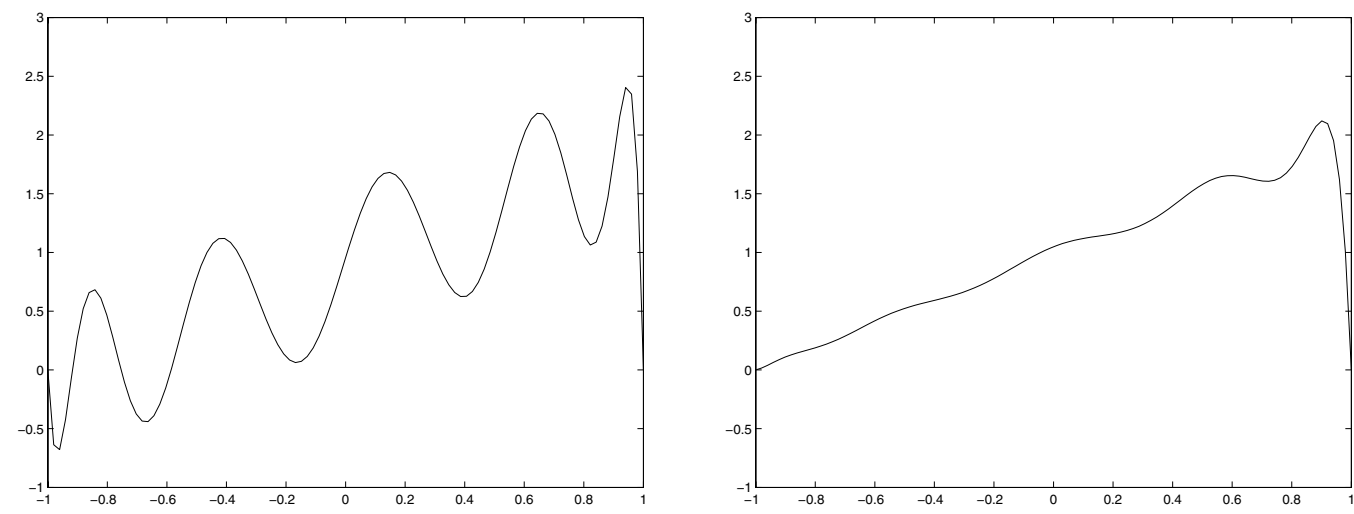

Figure 6. Approximated solution $u_{n}$ (left) and $w_{n}$ (right) of problem (28) with $f=1$, $\epsilon=0.001, \beta=1$ and $n=11$ obtained with standard collocation (left) and superconsistent collocation (right) respectively.

TABLE 5. Comparison of the maximum preconditioned eigenvalues in the case of the advectiondiffusion equation when $\beta=1$.

\begin{tabular}{cccc}
\hline $\mathrm{n}$ & $\epsilon$ & Collocation at $\mathbf{x}$ & Collocation at $\mathbf{z}$ \\
\hline 8 & & 2.125 & 1.952 \\
16 & & 2.297 & 2.187 \\
24 & 1 & 2.355 & 2.275 \\
32 & & 2.383 & 2.321 \\
64 & 2.426 & 2.392 \\
\hline 8 & & 3.429 & 1.001 \\
16 & & $2.165+0.696 \mathrm{i}$ & 2.013 \\
24 & 0.1 & $2.148+0.540 \mathrm{i}$ & 2.170 \\
32 & & $2.182+0.431 \mathrm{i}$ & 2.253 \\
64 & & 2.323 & 2.372 \\
\hline 8 & & 14.240 & $1.546+0.071 \mathrm{i}$ \\
16 & & $2.594+1.320 \mathrm{i}$ & 1.823 \\
24 & 0.05 & $2.323+1.164 \mathrm{i}$ & 2.009 \\
32 & & $2.256+0.970 \mathrm{i}$ & 2.126 \\
64 & & $2.279+0.549 \mathrm{i}$ & 2.322 \\
\hline 8 & & negative real part & $1.410+0.030 \mathrm{i}$ \\
16 & & 134.15 & $1.542+0.035 \mathrm{i}$ \\
24 & 0.01 & $5.362+3.427 \mathrm{i}$ & 1.658 \\
32 & & $4.148+3.828 \mathrm{i}$ & 1.746 \\
64 & $2.760+2.823 \mathrm{i}$ & 2.045 \\
\hline
\end{tabular}

For a given function $v$ vanishing at $x= \pm 1$, the $i$-th component, $i=1, \ldots, n-1$, of the vector obtained by applying $M_{n-1}$ to the vector $v(\mathbf{x})$ is given by:

$$
\begin{aligned}
\left\{M_{n-1} v(\mathbf{x})\right\}_{i}= & \frac{-2 \epsilon}{x_{i+1}-x_{i-1}}\left[\frac{v\left(x_{i+1}\right)-v\left(x_{i}\right)}{x_{i+1}-x_{i}}-\frac{v\left(x_{i}\right)-v\left(x_{i-1}\right)}{x_{i}-x_{i-1}}\right] \\
& +\beta\left[v\left(x_{i-1}\right) \frac{2 z_{i}-x_{i}-x_{i+1}}{\left(x_{i-1}-x_{i}\right)\left(x_{i-1}-x_{i+1}\right)}+v\left(x_{i}\right) \frac{2 z_{i}-x_{i-1}-x_{i+1}}{\left(x_{i}-x_{i-1}\right)\left(x_{i}-x_{i+1}\right)}\right. \\
& \left.+v\left(x_{i+1}\right) \frac{2 z_{i}-x_{i}-x_{i-1}}{\left(x_{i+1}-x_{i-1}\right)\left(x_{i+1}-x_{i}\right)}\right]
\end{aligned}
$$



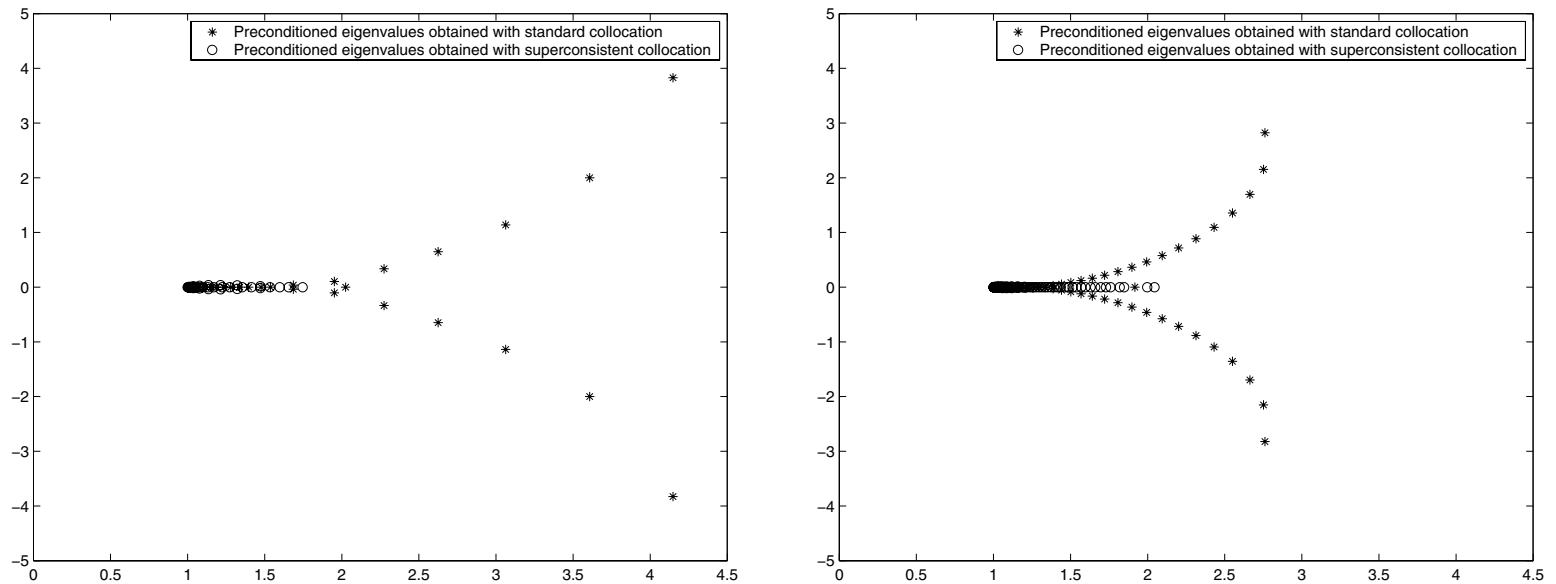

FIGURE 7. Spectra of the preconditioned matrices obtained by standard and superconsistent collocation respectively, when $\epsilon=0.01, \beta=1$ and $n=32$ (left), $n=64$ (right).
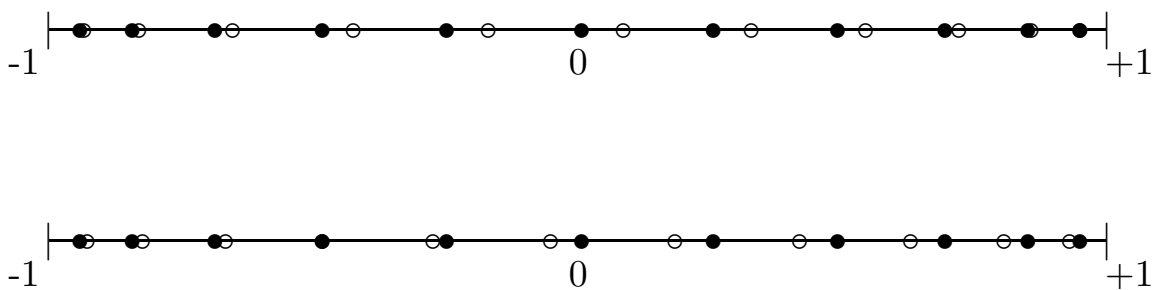

FIGURE 8. The grid $\mathbf{x}$ (black circles) and the grid $\mathbf{z}$ (white circles) for the superconsistent Chebyshev method with $n=12, \epsilon=0.05$ and $\beta(x)=x^{2}-1$ (top), $\beta(x)=x+0.5$ (bottom).

with $x_{0}=-1$ and $x_{n}=1$. Concerning the choice of the collocation points $z_{i}, i=1, \ldots, n-1$, we used the same $\mathbf{z}$ recovered from the pseudospectral approximation. These nodes are the zeros of $T_{n}^{\prime}$ for the standard collocation method, or the zeros given by $(31)$, where $\chi_{n+1}=\left(1-x^{2}\right) T_{n}^{\prime}$, for the superconsistent method. An alternative would be to use the nodes making superconsistent the finite-difference approximation, which are obtained by looking for the zeros of $-\epsilon \chi_{3}^{\prime \prime}+\beta \chi_{3}^{\prime}$ where $\chi_{3}(x)=\left(x-x_{i-1}\right)\left(x-x_{i}\right)\left(x-x_{i+1}\right)$. This second choice is not as effective as the first one, so that it will be not implemented in the experiments below.

In Table 5 , for $\beta=1$ and different values of $n$ and $\epsilon$, we compare the maximum (i.e., the one with the highest complex norm) preconditioned eigenvalue, both in the standard collocation case and the superconsistent collocation case. Concerning the second method we observe a clear improvement, bringing to eigenvalues bounded with respect to both $\epsilon$ and $n$. In addition, Figure 7 shows the distribution in the complex plane of the eigenvalues for $\epsilon=0.01, \beta=1$ when $n=32$ and $n=64$. We used the symbol $*$ for the standard Chebyshev collocation and the symbol $\circ$ for the superconsistent collocation. It is worthwhile to remark that such computations have been carried out in double precision. Actually these kind of matrices are very sensitive to roundoff errors, as detailed in [20]. Thus, it is not excluded that extra care is needed in order to get more reliable results.

For the variable coefficient case the organization of the job is going to be more tricky, although we obtain perfectly similar results. The superconsistent nodes are shifted left or right according to the sign of the coefficient 

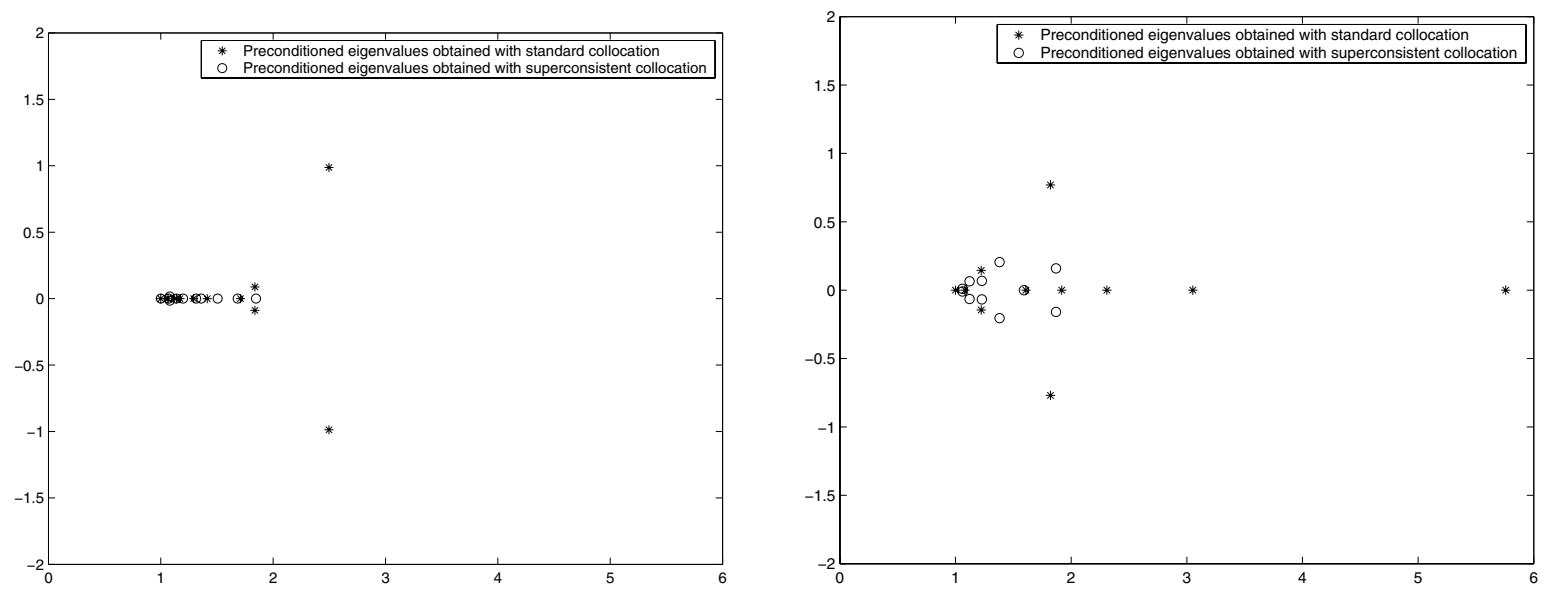

FIGURE 9. Spectra of the preconditioned matrices obtained by standard and superconsistent collocation respectively, when $n=12, \epsilon=0.05$ and $\beta(x)=x^{2}-1$ (left), $\beta(x)=x+0.5$ (right).

$\beta=\beta(x)$ in (28). Each collocation node $z_{i}, i=1, \ldots, n-1$, can be independently computed, according to the local direction and magnitude of the advective term $\beta$. Finally, the discretization matrix is assembled as in (33) and the preconditioner as in (34) where the coefficient $\beta$ is evaluated in $z_{i}, i=1, \ldots, n-1$. Hints for the implementation are also given in [7], where the Legendre superconsistent case with variable coefficients is also discussed. We provide here some results. Figure 8 shows, for $n=12, \epsilon=0.05, \beta(x)=x^{2}+1$ and $\beta(x)=x+0.5$, the distribution of the nodes $z_{i}, i=1, \ldots, 11$ (white circles) and the one of the nodes $x_{i}, i=1, \ldots, 11$ (black circles). The displacement in the complex plane of the corresponding eigenvalues is shown in Figure 9, where we used the symbol $*$ for the standard Chebyshev collocation and the symbol $\circ$ for the superconsistent collocation. Note that, in the second case, $\beta$ changes sign in $]-1,1[$. Therefore, both -1 and +1 are outflow boundary points. The corresponding collocation nodes $z_{i}, i=1, \ldots, n-1$ are of upwind type, thus, as one can see in Figure 8, part of them is shifted on the right and the remaining is shifted on the left.

\section{REFERENCES}

[1] C. Canuto and P. Pietra, Boundary interface conditions within a finite element preconditioner for spectral methods. J. Comput. Phys. 91 (1990) 310-343.

[2] C. Canuto and A. Quarteroni, Spectral and pseudo-spectral methods for parabolic problems with nonperiodic boundary conditions. Calcolo 18 (1981) 197-218.

[3] C. Canuto, M.Y. Hussaini, A. Quarteroni and T.A. Zang, Spectral Methods in Fluid Dynamics. Springer, New York (1988).

[4] L. Fatone, D. Funaro and G.J. Yoon, A convergence analysis for the superconsistent Chebyshev method. Appl. Num. Math. (2007) (to appear).

[5] D. Funaro, Polynomial Approximation of Differential Equations, Lecture Notes in Physics 8. Springer, Heidelberg (1992).

[6] D. Funaro, Some remarks about the collocation method on a modified Legendre grid. J. Comput. Appl. Math. 33 (1997) 95-103.

[7] D. Funaro, Spectral Elements for Transport-Dominated Equations, Lecture Notes in Computational Science and Engineering 1. Springer (1997).

[8] D. Funaro, A superconsistent Chebyshev collocation method for second-order differential operators. Numer. Algorithms 28 (2001) 151-157.

[9] D. Funaro, Superconsistent discretizations. J. Scientific Computing 17 (2002) 67-80.

[10] D. Gottlieb, M.Y. Hussaini and S.A. Orszag, Theory and application of spectral methods, in Spectral Methods for Partial Differential Equations, R.G. Voigt, D. Gottlieb and M.Y. Hussaini Eds., SIAM, Philadelphia (1984).

[11] P. Haldenwang, G. Labrosse, S. Abboudi and M. Deville, Chebyshev 3-D spectral and 2-D pseudospectral solvers for the Helhmoltz equation. J. Comput. Phys. 55 (1981) 115-128.

[12] T. Kilgore, A characterization of the Lagrange interpolation projections with minimal Tchebycheff norm. J. Approximation Theory 24 (1978) 273-288. 
[13] D.H. Kim, K.H. Kwon, F. Marcellán and S.B. Park, On Fourier series of a discrete Jacobi-Sobolev inner product. J. Approximation Theory 117 (2002) 1-22.

[14] S.D. Kim and S.V. Parter, Preconditioning Chebyshev spectral collocation method for elliptic partial differential equations. SIAM J. Numer. Anal. 33 (1996) 2375-2400.

[15] S.D. Kim and S.V. Parter, Preconditioning Chebyshev spectral collocation by finite-difference operators. SIAM J. Numer. Anal. 34 (1997) 939-958.

[16] F. Marcellán, B.P. Osilenker and I.A. Rocha, Sobolev-type orthogonal polynomials and their zeros. Rendiconti di Matematica 17 (1997) 423-444.

[17] E.H. Mund, A short survey on preconditioning techniques in spectral calculations. Appl. Num. Math. 33 (2000) 61-70.

[18] S.A. Orszag, Spectral methods for problems in complex geometries. J. Comput. Phys. 37 (1980) 70-92.

[19] G. Szegö, Orthogonal Polynomials. American Mathematical Society, New York (1939).

[20] L.N. Trefethen and M. Embree, Spectra and Pseudospectra: the behavior of nonnormal matrices and operators. Princeton University Press (2005). 